\title{
ESTUDIO SOCIOLINGÜÍSTICO DE LA INTERDENTALIZACIÓN DE /k/, DENTRO DEL GRUPO /kt/, EN EL ESPAÑOL HABLADO EN LA CIUDAD DE SANTANDER (ESPAÑA)
}

\author{
Jaime Peña Arce
}

Universidad Complutense de Madrid

\begin{abstract}
RESUMEN: El objetivo de este trabajo es analizar, desde una perspectiva diastrática y diafásica, una realidad - la interdentalización de /k/, dentro del grupo /kt/- señalada ya, desde una perspectiva diatópica (Alvar, 1995; Fernández Juncal, 2001; Moreno Fernández, 2009), como propia del español de Cantabria. Para ello, y centrada en la ciudad de Santander, capital de la región, se realizó una encuesta a 77 informantes, respetando las variables de edad, sexo y nivel sociocultural en relación con las características de esa población. Los resultados evidencian la extensión de la articulación interdentalizada en el mencionado contexto fónico, aunque con grandes diferencias según el nivel sociocultural del hablante y el registro, y no tanto según el género o la edad.
\end{abstract}

PALABRAS CLAVE: fonética, sociolingüística, interdentalización, español de Cantabria, grupos consonánticos

Sociolinguistic study of the interdentalization of $/ \mathrm{k} /$, in the cluster $/ \mathrm{kt} /$, in the Spanish spoken in the city of Santander (Spain)

\begin{abstract}
The interdentalization of / $\mathrm{k}$ / in the cluster / $\mathrm{kt} /$ has been studied geographically by Alvar (1995), Fernández Juncal (2001) and Moreno Fernández (2009), who concluded that it was characteristic of Spanish spoken in Cantabria. The purpose of this paper is to study this same phenomenon from a stratificational and situational perspective, but only in relation to the city of Santander. A sample of 77 informants that accurately reflected the demographics of the city as regards sex, age and education was identified. These informants were then interviewed so as to elicit from them examples of both spontaneous and monitored speech. The results show that interdentalization of $/ \mathrm{k} /$ in the / $\mathrm{kt} /$ cluster covaries, in Santander, with level of education and register, but not with age or sex.
\end{abstract}

KEYWORDS: phonetics, sociolinguistics, interdentalization, Spanish spoken in Cantabria, consonant clusters

\section{INTRODUCCIÓN}

El presente trabajo sigue el modelo y los métodos de investigación de la sociolingüística variacional (Labov, 1966; Shuy, Wolfram y Riley, 1968; Labov, 1972a, 1972b; Trudgill, 1974; 
Cedergren, 1983; Silva-Corvalán, 1989). Los objetivos que pretendemos alcanzar son los siguientes: 1) determinar la vitalidad de la articulación interdental fricativa sorda [ $\theta]$, dentro del grupo consonántico / kt/, en detrimento de otras soluciones alofónicas, dadas en ese contexto, dentro de las hablas santanderinas; 2) relacionar el uso de esas variaciones alofónicas con factores sociales y contextuales dentro del mencionado espacio geográfico; y 3) identificar posibles tendencias de cambio lingüístico en el español actual, en especial en el de la zona septentrional de la Península y, concretamente, en el empleado en la Comunidad Autónoma de Cantabria.

Para ello, este estudio se ha dividido en dos grandes apartados: por un lado, un estado de la cuestión, en el que se presenta, en primer lugar y desde una dimensión histórica, el proceso de cambio y variación que ha sufrido en nuestra lengua el grupo culto latino /kt/; a continuación, se describe el proceso de interdentalización de / $/$ / desde una perspectiva articulatoria y se sintetiza la repercusión de esta variación fonética - gracias a los estudios existentes- desde una dimensión diatópica, con matices diastráticos y diafásicos; este apartado finaliza, a modo de introducción a la investigación principal, con un retrato del estado de esta variación en las hablas montañesas, desde un prisma únicamente geográfico, para lo cual se utilizan los datos del Atlas Lingüístico y Etnográfico de Cantabria (1995). Concluido el estado de la cuestión, se presenta, en el segundo apartado, la encuesta realizada, con diversas consideraciones sobre la metodología empleada en su creación y las características que la vertebran para, acto seguido, dar a conocer -desglosados según una serie de variables sociales y con una permanente atención al registro- los datos extraídos de ella. Para facilitar la comprensión, se incluye una serie de tablas que ofrecen al lector los datos sintetizados.

\section{ESTADO DE LA CUESTIÓN}

2.1. El grupo culto /kt/. Procesos de cambio y variación: la interdentalización de /k/

El grupo latino /kt/ sufrió, en el tránsito del latín al español, un proceso de cambio, articulado en torno a los siguientes estados evolutivos: $/ \mathrm{kt} />[\mathrm{xt}]>/ \mathrm{it} />\widehat{\mathrm{t}} \mathrm{f} /$, que forzó su transformación en el fonema postalveolar africado sordo. Pese a ser esta una tesis universalmente aceptada, existen aún diferentes puntos de vista sobre la intrahistoria de esta evolución. Estas divergencias evidencian la existencia de una abundante variación alofónica (García García de León, 2015) dentro de una cronología difícil de determinar con exactitud.

Sea como fuere el proceso de cambio en diacronía, puede afirmarse que la pervivencia del grupo /kt/ en el español contemporáneo queda restringida a las voces cultas y semicultas que, o bien no sufrieron una evolución patrimonial, o bien fueron rescatadas de la lengua madre en algún momento posterior a la época de los orígenes de nuestro idioma. Como consecuencia natural, /kt/ se constituyó, desde su reincorporación, como un grupo altamente inestable, sujeto -siempre que no intervengan especiales factores de énfasis en 
su articulación- a diferentes procesos de variación (García García de León, 2015); dentro de estos, es el fenómeno de la interdentalización del fonema implosivo / $\mathrm{k} /$ el que interesa en este estudio.

\subsubsection{La interdentalización de /k/}

Según Fernández Sevilla, "la mayoría de los grupos cultos aparecen, por lo general, en vocablos también de carácter culto, lo que facilita su conservación” (1990, 474). No obstante, en la lengua oral de las personas instruidas, y dentro de un contexto familiar o poco formal, su articulación tiende a relajarse y simplificarse (Fernández Sevilla, 1990, 474). Es precisamente en ese contexto, y siempre que las voces gocen de vitalidad, donde aflora el fenómeno de la interdentalización de /k/ dentro del grupo /kt/. En la bibliografía existente relacionada con el análisis de este proceso, han primado los estudios de carácter articulatorio, pues los de carácter acústico ${ }^{1}$ no han ofrecido conclusiones relevantes (Fernández Planas y Martínez Celdrán, 2008, 63).

Así las cosas, desde una perspectiva articulatoria, la transformación de /k/ en / $\theta /$, que da lugar a pronunciaciones del tipo de arquitezto o viztoria, viene determinada por dos procesos: el primero afectaría al punto de articulación, que experimentaría un adelantamiento (de velar a interdental), y el segundo modificaría el modo de articulación, con el paso de una articulación oclusiva a una fricativa. Ya Navarro Tomás (1918, § 127), pese a no mencionar de manera explícita la interdentalización, señaló, con relación al cambio del modo de articulación de /k/ dentro del grupo /kt/, lo siguiente:

Conviene advertir que en el grupo $c t$ la $c$ se pronuncia solamente implosiva, sin explosión perceptible; además, la lengua, para articular la $c$, solo llega de ordinario a formar una verdadera oclusión un instante antes de pasar a la posición de la $t$ siguiente, resultando, por tanto, fricativa, en la conversación corriente, una gran parte de dicha c; claro es que en pronunciación fuerte esa fricación desaparece y la $c$ resulta completamente oclusiva.

El filólogo manchego se limitaba a agregar seguidamente, y a pie de página, que "la pronunciación vulgar presenta diferentes variantes del grupo ct: dotor, caráiter, aspeuto, aztor, fastor, etc.”. Más adelante, este mismo investigador, en la descripción que ofrecía sobre los enlaces de los segmentos dentro de los grupos consonánticos heterosilábicos, afirmaba que:

En el grupo de dos oclusivas sordas $(p t, c t)$, la primera es implosivo-oclusiva y la segunda oclusivo-explosiva; durante la oclusión, los órganos pasan de la primera consonante a la segunda, sin que la transición sea acústicamente perceptible; la división silábica recae, pues, sobre dicha oclusión...; la primera consonante en este caso es, en general, una articulación relajada que a veces se reduce a fricativa y a veces se pierde totalmente en la pronunciación, aun cuando, como ocurre en septiembre, etc., siga conservándose en la escritura (1918, § 149). 
Llorente fue el primero en describir el proceso de la interdentalización de / $\mathrm{k} /$ - en su opinión, un fenómeno de economía articulatoria que vendría a paliar la difícil pronunciación del grupo /kt/-, y lo hizo de la siguiente manera: "la implosiva velar se interdentaliza, acercando así su punto de articulación al de la $t$, al mismo tiempo que se hace fricativa para favorecer su transición” (1947, 109). Según este especialista, la interdentalización sería un fenómeno moderno, frente a la pérdida de la velar (dotor por doctor) o su vocalización (aspeuto por aspecto), soluciones conocidas ya desde la época clásica. Llorente califica la interdentalización como un proceso de cambio de "raigambre leonesa" $(1947,109)$ y relativamente reciente en el tiempo, aunque no fija una cronología precisa sobre su génesis, implantación y extensión.

La Fonética y Fonología de la NGLE indica, de manera general, que el español, como resultado espontáneo de su evolución desde el latín, no presenta alófonos oclusivos en posición de coda silábica, a excepción de los cultismos, y que la tendencia general de la lengua lleva, en estos casos, a procesos de pérdida de rasgos o -directamente- a su elisión (RAE y ASALE, 2011, § 4.7g). Más adelante, y en alusión concreta a la consonante velar en posición implosiva, señala que esta pierde alguno de sus rasgos (RAE y ASALE, 2011, § 4.70) en el mencionado contexto fónico.

En definitiva, parece que, articulatoriamente, el fonema implosivo -siempre que no desaparezca- se debilita, lo que hace que pierda alguno de sus rasgos característicos. Estos rasgos son, dentro del proceso de la interdentalización de /k/ en el seno del grupo /kt/, dos: el primero afecta al modo de articulación, donde la oclusión pierde tensión y el sonido se convierte en fricativo; y el segundo se centra en el punto de articulación, que - por ser la / $\mathrm{t}$ / una consonante dental- se adelanta para acercarse a esta, por lo que la realización pasa de ser velar a interdental. Así, la realización de una $[\theta]$ en lugar de una $/ \mathrm{k} /$ sería una forma ultracorrecta de articular el grupo culto /kt/, preteriendo la elisión, considerada una solución marcadamente vulgar.

2.2. Extensión geográfica de la interdentalización de /k/ dentro del grupo /kt/. Matices sociolingüísticos y contextuales

Con lo visto hasta ahora, cabe afirmar que, sobre el tratamiento del grupo /kt/ en el español, puede realizarse -además de una aproximación histórica- un análisis variacionista, tanto desde una perspectiva diatópica y diastrática, tal como las definió Flydal (1952, 244-251), como diafásica o contextual, según la formulación de Coseriu (1981, 12). La primera referencia sobre la localización geográfica de la interdentalización de $/ \mathrm{k} /$, dentro del grupo /kt/, la aporta -como cabría esperar-Llorente, quien afirma que esta es una característica del habla salmantina. Tras la perspectiva diatópica, incluye observaciones de carácter diastrático - "también se encuentra en castellano vulgar, sobre todo en el lenguaje de las clases bajas de Madrid y Valladolid" $(1947,109)$ - y diafásico - “esta solución... no solo es empleada por la gente de mediana instrucción, sino que en 
toda esta zona de Salamanca y Valladolid se usa aún en la conversación familiar y descuidada de personas cultas" (1947, 109-110)-.

Tras esta primera alusión, son multitud las menciones a este proceso que encontramos en la bibliografía. Lapesa $(1981, \S 118)$ describió este fenómeno como general en todo el castellano septentrional, y apuntó que se daba, muy especialmente, en la pronunciación cuidada que rehuía la elisión. Neira Martínez (1976) señalaba la presencia de articulaciones interdentalizadas tanto en el español de Asturias como en el bable, y en 1998 Antón confirmó la presencia de la interdentalización como una de las soluciones más habituales en el tratamiento de / $\mathrm{k}$ / oclusiva postnuclear en el habla de Langreo. Fue, nuevamente, Llorente $(1965,328)^{2}$ quien retomó la cuestión y extendió el fenómeno a las hablas riojanas. Ese mismo año, Quilis $(1965,23)$ apuntó que la solución interdentalizada era común en Madrid, pero no entre la gente de mayor edad. González Ferrero (1986, 205) describió, a mediados de los ochenta, este proceso en el habla de la comarca zamorana de Aliste. También en el decenio de 1980 se realizaron sendos estudios sociolingüísticos sobre el habla de las ciudades de Burgos y Valladolid: en el caso de la capital burgalesa, la solución interdental para el grupo /kt/ era, con mucho, la mayoritaria (Martínez Martín, 1983, 143); en la capital vallisoletana ocurría lo mismo (Williams, 1987, 103-110). ${ }^{3}$ Alonso Hernández (1996, 201) sentenció que, si bien esta era una variación generalizada en todo el norte peninsular, era en las provincias de Salamanca, Zamora, Valladolid y Ávila donde más se repetía. Alvar, en su Atlas Lingüístico y Etnográfico de Castilla y León (1999), dedicó numerosos mapas al estudio de esta cuestión: un vistazo, por ejemplo, al mapa n. ${ }^{\circ} 40$, recto, - $a$, muestra la extensión de la articulación fricativa e interdentalizada, que alcanza, por provincias, los siguientes porcentajes: Palencia (95 \%), Valladolid (94\%), Zamora (90 \%), Salamanca (86 \%), Segovia (80 \%), León (78.5 \%), Burgos (78 \%), Soria (70 \%) y Ávila (68 \%); finalmente, Borrego Nieto (2001) calificó la interdentalización de /k/ como la única particularidad fónica característica a toda la comunidad castellanoleonesa. En el último lustro del siglo pasado, Moreno Fernández $(1996,216)$ reseñó la presencia de esta variación fonética en todas las provincias de Castilla-La Mancha, ${ }^{4}$ especialmente en Guadalajara y en Cuenca. Respecto a la situación en Cantabria, Fernández Juncal, que ha dedicado numerosas obras a la variación dialectal montañesa (1998, 2000, 2001 o 2009, entre otras), reseñó este fenómeno en el español de la zona oriental de esta región (2001, 31); en la misma dirección, Moreno Fernández (2009, 134-135) defendió el carácter general de la articulación interdentalizada del grupo /kt/ en las hablas de Cantabria.

En conclusión, parece que nos encontramos ante un fenómeno de matriz leonesa, surgido en las provincias de Salamanca y Zamora, irradiado ya por toda Castilla y León, y que ha desbordado los límites, por el sur, del Sistema Central, alcanzando a Madrid y a Castilla-La Mancha; por el norte, de la Cordillera Cantábrica, con manifestaciones en las autonomías del Cantábrico Central; y por el noreste, más allá del Sistema Ibérico, con evidencias en el curso alto del Ebro. Además, el fenómeno no tendría solo una dimensión diatópica, sino que - para su correcta aquilatación- sería necesario abordarlo también desde una perspectiva diastrática y diafásica. 


\subsection{La situación en el español de Cantabria según los datos del ALECant}

Antes de presentar la encuesta, aportación principal del presente estudio, y a modo de aproximación a la cuestión analizada en el habla de la ciudad de Santander, se ofrece una perspectiva geográfica, a partir de datos espigados del Atlas Lingüístico y Etnográfico de Cantabria, sobre la extensión de la interdentalización de la consonante velar, dentro del grupo /kt/, en el español de la región montañesa. Para ello, han sido analizados cuatro mapas de esa obra - el n. ${ }^{\circ} 851$, eructar; el n. ${ }^{0} 1071$, recta; el n. ${ }^{\circ} 1072$, efecto; y el n. ${ }^{\circ}{ }^{1073}$, octubre- y sus datos han sido cotejados entre sí. Los resultados se ofrecen en forma de mapa (véase a continuación) para que el acercamiento a la cuestión sea rápido y sencillo:

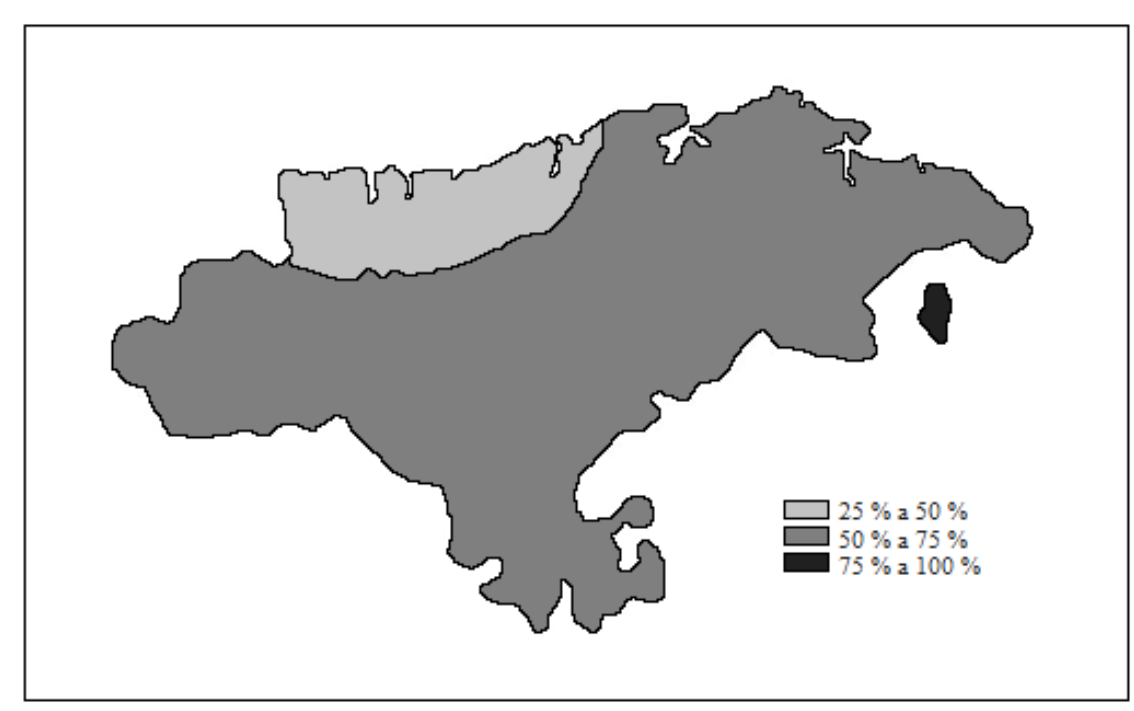

Mapa 1. Distribución geográfica de la interdentalización de /k/ en Cantabria, según los datos del ALECant

Los datos del Atlas de Cantabria muestran que el porcentaje más bajo, aunque nunca inferior al $40 \%$, de la solución [ $\theta$ ] para el primer elemento del grupo /kt/ se da en la zona costera occidental, desde la desembocadura del río Pas hasta el límite con los concejos asturianos más orientales. La mayor parte del territorio montañés presenta porcentajes de entre un $50 \%$ y un $75 \%$ de interdentalización en este contexto: se acercan más a esta última cifra las zonas montañosas de la Liébana (zona suroccidental), los valles centrales de la cuenca del río Besaya, la meridional comarca de Campoo (situada ya al sur de la divisoria de aguas de la Cordillera Cantábrica), los valles del alto Pas, el entorno de la bahía de Santander y la costa occidental; en las zonas interiores de la Trasmiera (área centrooriental), en los valles de Soba y Ruesga, en el curso medio del Pas y en la zona baja del valle de Liébana, la incidencia de la interdentalización es algo menor, aunque superior al $50 \%$. Por último, es de destacar que en el Valle de Villaverde, un enclave situado en el corazón de las Encartaciones vizcaínas, la articulación fricatizada y adelantada alcanza porcentajes cercanos al $100 \%$. 


\section{MuESTRA}

3.1. Características y metodología de la muestra

3.1.1. Características de la muestra. Sexo, edad y nivel sociocultural de los informantes

La encuesta, que trata de determinar el grado de extensión y la distribución social y contextual de la solución interdentalizada -que sustituye a la realización canónica de la velar oclusiva sorda posnuclear, en el seno del grupo /kt/- en el español hablado en Santander, capital de la Comunidad Autónoma de Cantabria, ha incluido a 77 informantes (elegidos de forma aleatoria) que, dentro de una población de 173721 personas, 5 equivale al $0.045 \%$. Este volumen de participantes se ha considerado más que satisfactorio si se compara con los 60 que seleccionó Trudgill (1974) de un total de 118610 habitantes -o con los 110 de Perissinotto (1975) sobre una población superior a los siete millones de habitantes, o con los 105 de López Morales (1983) para una población de algo menos de medio millón-. En la selección de estos 77 informantes no se tuvo en cuenta a las comunidades que no pueden formar parte de un estudio de este tipo: los menores de 20 años, por encontrarse aún en un periodo formativo (este grupo representa el $16 \%$ de los habitantes de la ciudad $)^{6}$ y los inmigrantes, que representan el $7 \%^{7}$ de los habitantes de Santander.

Los requisitos para poder ser informante han sido cinco:

1. Haber nacido o ser residente en la ciudad de Santander desde los seis años de edad.

2. Haber vivido toda su vida en esta ciudad.

3. Ser hijo de españoles - preferentemente, de santanderinos-.

4. Haber recibido toda su formación escolar y/o académica, por breve o larga que fuera, en la propia ciudad de Santander.

5. Los informantes no podían padecer ninguna patología relacionada con el habla.

Las variables sociales tenidas en cuenta en este trabajo han sido tres: el sexo de los informantes, su edad y su nivel sociocultural.

1) Sexo: a partir de los datos sobre el componente poblacional femenino (54\% del total) y masculino ( $46 \%$ del total) de la ciudad, ${ }^{8}$ se decidió seleccionar aleatoriamente - para ser respetuoso con estos porcentajes- a 43 informantes mujeres y a 34 hombres.

2) Edad: se ha dividido a los informantes, desde una perspectiva émica9 (Eckert, 1997), en tres grupos de edad: a) de 20 a 34, b) de 35 a 54 y c) de más de 55 . Gracias a los datos de la pirámide poblacional de la ciudad ${ }^{10}$ - según la cual, la cifra de población de entre los 20 y los 34 años es de 26184 (un $15 \%$ del total); la de población entre los 35 y los 54 años, 50284 (un 29 \% del total); y la de población 
mayor de 55 años, de 69431 (un $40 \%$ del total)- fueron aleatoriamente seleccionados 14 informantes del grupo a), 25 del grupo b) y 38 del grupo c).

3) Nivel sociocultural: se ha dividido a los participantes en tres grupos. El primero (nivel bajo) incluye a aquellos colaboradores que no poseían el graduado escolar -dentro de los cuales el porcentaje de analfabetos era residual-; el segundo (nivel medio) agrupa a los que sí habían conseguido ese graduado o habían completado los estudios secundarios; y el tercero (nivel alto) engloba a las personas que habían conseguido un título universitario. Merced a los datos de la distribución de la población santanderina según su nivel de instrucción ${ }^{11}$ (36 288 habitantes sin el graduado, un $18 \%$ del total; 100305 con el graduado o el título de secundaria, un $45 \%$ del total, y 37128 universitarios, un $18 \%$ del total), la selección aleatoria de los informantes ha sido: 14 colaboradores sin el graduado - una de ellas, analfabeta-, 45 informantes con el título de educación secundaria y 18 con una titulación universitaria.

\subsubsection{Metodología de la muestra y extracción de los ejemplos}

Siguiendo las normas de la sociolingüística variacionista, se realizaron -entre el 21 de enero y el 5 de mayo del 2019- entrevistas grabadas con los informantes; estos pudieron elegir, en todo momento, el lugar donde querían que la recogida de datos se llevase a cabo: sus hogares, sus lugares de trabajo u otros locales a su elección, como casas de amigos o espacios públicos. Cada grabación contiene de unos quince a veinte minutos de conversación informal parcialmente dirigida, que el informante entendía como externa al trabajo, así como una lectura ${ }^{12}$ de un texto periodístico de temática política -relacionado con el ciclo electoral español, vivido en la primera mitad del año en curso (2019)-, que servía de transición para una segunda lectura, esta vez de una lista de palabras que contenían el mencionado grupo consonántico. El objetivo de esta variedad de procedimientos era forzar la creación de distintos registros contextuales, más coloquiales (conversación informal) y más formales (lecturas dirigidas), que permitieran un estudio general de la cuestión en cuanto a su dimensión diafásica.

Puesto que el interés de este estudio reside en la distribución social y contextual de la articulación interdentalizada del fonema velar oclusivo de la de secuencia /kt/, no se ha estudiado en profundidad toda la variación alofónica que los resultados de la muestra han ofrecido; solo se han considerado, por tanto, las tres soluciones mayoritarias: la realización interdental $[\theta]$, el mantenimiento de la articulación velar oclusiva sorda [k], y la elisión del primer miembro del grupo [ø]. El corpus total del estudio quedó conformado, tras escuchar atentamente las grabaciones y desechar los ejemplos que no correspondían a las tres soluciones antes mencionadas, por 3817 casos. El estudio de todas las realizaciones, tanto las descartadas (287) como las incluidas (3817), fue realizado mediante el programa informático Praat. 


\subsection{Resultados de la muestra y análisis de sus particularidades}

Los resultados del análisis de los 3817 ejemplos estudiados se presentan en cuatro epígrafes: el primero incluye los datos totales, y los tres siguientes el desglose según las variables sociales sexo, edad y nivel sociocultural. En todos los casos, y en respuesta al interés manifestado por profundizar en la dimensión diafásica del fenómeno, se hará referencia al registro en el que se produjo la articulación: relajado o formal.

\subsubsection{Resultados totales}

Los resultados globales, extraídos a partir del estudio de los 3817 casos que conforman el corpus de esta investigación, se muestran en la tabla 1:

\begin{tabular}{|l|c|c|}
\hline \multicolumn{1}{|c|}{ Articulación } & N. $^{\text {o de ejemplos }}$ & Porcentaje \\
\hline Oclusiva velar sorda [k] & 929 & $24.3 \%$ \\
\hline Fricativa interdental sorda $[\theta]$ & 1897 & $49.7 \%$ \\
\hline Cero fonético [ø] & 991 & $26 \%$ \\
\hline
\end{tabular}

Tabla 1. Resultados de la articulación de /k/ dentro del grupo /kt/ en la comunidad de habla santanderina

Con estos datos, puede afirmarse que -independientemente del registro (relajado o formal) en el que se produjo la articulación- la articulación interdentalizada es la mayoritaria, pues alcanza el $49.7 \%$ de los ejemplos del corpus; la segunda solución con mayor prevalencia, y a mucha distancia, sería la elisión (26 \% del total); el mantenimiento de la articulación velar [k] le iría a la zaga, con el $24.3 \%$ del total. Estos datos vendrían a coincidir, cuantitativamente, con los aportados para el Atlas de Cantabria para la región montañesa (mapa 1), pues ambos estudios coinciden en señalar como mayoritaria la solución [ $\theta$ ]; sin embargo, los resultados del ALECant mostraban una estimación de la presencia de la solución interdentalizada de en torno al 50-75 \%, claramente superior a la que arroja la presente investigación.

Por otro lado, los datos totales según el registro - relajado o formal- son los siguientes (tabla 2):

\begin{tabular}{|c|c|c|c|c|c|c|}
\hline \multirow{2}{*}{ Registro } & \multicolumn{5}{|c|}{ Tipos de articulación } \\
\cline { 2 - 7 } & \multicolumn{2}{|c|}{$[\mathrm{k}]$} & \multicolumn{2}{|c|}{$[\theta]$} & \multicolumn{2}{c|}{$[\varnothing]$} \\
\cline { 2 - 7 } & $\mathrm{N.}^{\mathrm{o}}$ & Porc. & $\mathrm{N}^{\circ}$ & Porc. & N. ${ }^{\mathrm{O}}$ & Porc. \\
\hline Relajado & 286 & $14.3 \%$ & 963 & $48.5 \%$ & 742 & $37.2 \%$ \\
\hline Formal & 643 & $35.2 \%$ & 934 & $51.1 \%$ & 249 & $13.7 \%$ \\
\hline
\end{tabular}

Tabla 2. Resultados de la articulación de /k/ dentro del grupo /kt/ en la comunidad de habla santanderina según el registro 
Tal como puede comprobarse, tanto en un registro formal (51.1\%) como en uno relajado (48.5 \%), el sonido interdental es el mayoritario, a mucha distancia de los demás. Dentro del registro formal, la articulación oclusiva velar sorda alcanza un porcentaje del $35.2 \%$; muy descolgada, con un $13.7 \%$, aparece el cero fonético. En el informal, tras la solución [ $\theta$ ], la elisión aparece en un respetable 37.2 \% de los casos; la articulación canónica se da solo en el $14.3 \%$ de los ejemplos.

\subsubsection{Resultados por sexo}

Los resultados según la variable social hombre/mujer, con referencias al registro -relajado o formal- en el que se produjo la articulación de las diferentes soluciones, se muestran en la tabla 3:

\begin{tabular}{|c|c|c|c|}
\hline \multirow{2}{*}{ Sexo } & \multicolumn{3}{|c|}{ Tipos de articulación y registro 13} \\
\cline { 2 - 4 } & {$[\mathrm{k}]$} & {$[\theta]$} & {$[\varnothing]$} \\
\hline \multirow{2}{*}{ Hombres } & R: $11.1 \%$ & R: $65.1 \%$ & R: $23.8 \%$ \\
& F: $25.5 \%$ & F: $61.3 \%$ & F: $13.2 \%$ \\
\hline \multirow{2}{*}{ Mujeres } & R: $11.3 \%$ & R: $64.9 \%$ & R: $23.8 \%$ \\
& F: $27.4 \%$ & F: $60.2 \%$ & F: $13.4 \%$ \\
\hline
\end{tabular}

Tabla 3. Resultados de la articulación de /k/ dentro del grupo / kt/ en la comunidad de habla santanderina según el sexo de los informantes y el registro

La variable sexo no arroja muchas diferencias. La incidencia en la frecuencia de la solución velar oclusiva sorda es bastante limitada tanto entre los hombres como entre las mujeres, aunque es dos puntos superior en el caso de estas últimas dentro de los registros más formales. La articulación interdental es, con mucho, la más frecuente para ambos sexos, independientemente del registro. La elisión del fonema $/ \mathrm{k} /$ presenta porcentajes de aparición muy similares, al margen del sexo del informante. Así las cosas, puede afirmarse que la variable sexo no influye en este proceso de variación, tal como ocurría en el habla de la ciudad de Burgos (Martínez Martín, 1983, 149).

\subsubsection{Resultados por edad}

Las conclusiones sobre la incidencia de la interdentalización, según la edad y el registro (formal o relajado) en el que se produjo la articulación de las diferentes soluciones, pueden verse a continuación (tabla 4): 


\begin{tabular}{|c|c|c|c|}
\hline \multirow{2}{*}{ Edad } & \multicolumn{3}{|c|}{ Tipos de articulación y registro } \\
\cline { 2 - 4 } & {$[\mathrm{k}]$} & {$[\theta]$} & {$[\varnothing]$} \\
\hline \multirow{2}{*}{ De 20 a 34 años } & R: $11.3 \%$ & R: $65.5 \%$ & R: $23.2 \%$ \\
& F: $25.6 \%$ & F: $61.1 \%$ & F: $13.3 \%$ \\
\hline \multirow{2}{*}{ De 35 a 54 años } & R: $10.2 \%$ & R: $66.2 \%$ & R: $22.6 \%$ \\
& F: $25.6 \%$ & F: $61.6 \%$ & F: $12.8 \%$ \\
\hline \multirow{2}{*}{ Más de 55 años } & R: $11.8 \%$ & R: $64.4 \%$ & R: $23.8 \%$ \\
& F: $25.3 \%$ & F: $60.3 \%$ & F: $14.4 \%$ \\
\hline
\end{tabular}

Tabla 4. Resultados de la articulación de / k/ dentro del grupo / kt/ en la comunidad de habla santanderina según la edad de los informantes y el registro

La variable edad tampoco se muestra determinante. Dado el comportamiento casi idéntico de todos los grupos etarios, parece legítimo concluir que, con el discurrir del tiempo, la interdentalización de / $/$ / dentro del grupo / kt/ ha ido penetrando dentro del espacio geográfico del español septentrional. Al fin y al cabo, Quilis $(1965,23)$, en los años sesenta, afirmaba que $[\theta]$ no se daba entre los informantes mayores, y Martínez Martín (1983, 145), ya en los ochenta, informaba de que esta solución tenía, claramente, menor incidencia entre los hablantes mayores de 65 años. Sin embargo, en el caso de Santander, a finales del segundo decenio del siglo XXI, puede afirmarse que la diferencia entre generaciones es, prácticamente, inexistente.

\subsubsection{Resultados según el nivel sociocultural del hablante}

Los resultados a partir del nivel sociocultural del informante, en relación con el registro -formal o relajado - en el que se produjo la articulación de las distintas soluciones, son los que pueden verse en la tabla 5:

\begin{tabular}{|c|c|c|c|}
\hline \multirow{2}{*}{ Nivel sociocultural } & \multicolumn{3}{|c|}{ Tipos de articulación y registro } \\
\cline { 2 - 4 } & {$[\mathrm{k}]$} & {$[\theta]$} & {$[\varnothing]$} \\
\hline \multirow{2}{*}{ Nivel bajo } & R: $0.5 \%$ & R: $30.5 \%$ & R: $69 \%$ \\
& F: $1.4 \%$ & F: $63.8 \%$ & F: $34.8 \%$ \\
\hline \multirow{2}{*}{ Nivel medio } & R: $5.5 \%$ & R: $67.9 \%$ & R: $26.6 \%$ \\
& F: $25.5 \%$ & F: $64.9 \%$ & F: $9.6 \%$ \\
\hline \multirow{2}{*}{ Nivel alto } & R: $44.7 \%$ & R: $50.8 \%$ & R: $4.5 \%$ \\
& F: $84.7 \%$ & F: $11.1 \%$ & F: $4.2 \%$ \\
\hline
\end{tabular}

Tabla 5. Resultados de la articulación de /k/ dentro del grupo / kt/ en la comunidad de habla santanderina según el nivel sociocultural de los informantes y el registro 
La variable nivel sociocultural sí tiene una enorme incidencia en la cuestión analizada, más si se conjuga con el registro: los informantes de menor nivel sociocultural se decantan, en registros relajados, por la elisión de la consonante velar (69 \%); sin embargo, en registros formales, adoptan de forma mayoritaria la solución interdentalizada (63.8 \%). Los hablantes de un nivel medio se decantan, tanto en un registro relajado (67.9\%) como formal (64.9\%), por la variante interdentalizada, aunque esta tiene una incidencia algo superior en los registros relajados; dentro de este mismo grupo -y siempre por detrás de $[\theta]$ - la articulación [k] es la segunda más empleada en los registros formales, y la elisión es la segunda solución más habitual en un registro coloquial o familiar. Los hablantes con una formación universitaria prefieren, para un registro relajado, la variante interdentalizada (50.8 \%), seguida por la articulación de la oclusiva velar (44.7 \%); en registros formales, esta última solución es la hegemónica (84.7\%). Dentro del grupo alto, la elisión es excepcional, independientemente del registro.

Por tanto, la interdentalización resulta ser una solución propia del habla esmerada entre las clases bajas, que tienden a la elisión; natural entre la clase media, que rechaza la forma [k] en registros relajados y la elisión en los formales; y marcadamente familiar entre la clase alta. Esta última la emplea de forma mayoritaria -aunque en un porcentaje muy cercano al de la forma canónica- en ese registro, pero la elimina en los registros formales.

\section{CONCLUSIONES}

Tal como evidenciaban los datos del Atlas de Cantabria (1995), y como habían apuntado Fernández Juncal (2001, 31) y Moreno Fernández (2009, 134-135), parece que el fenómeno de la interdentalización de /k/, dentro del grupo /kt/, afecta de lleno al español hablado en la ciudad de Santander. Por otro lado, como también apuntaban los estudios y referencias sobre este fenómeno, un análisis meramente dialectal no resulta suficiente para conseguir una correcta aquilatación de este proceso de variación fonética en marcha. Los datos extraídos de la muestra que vertebra este trabajo corroboran esta afirmación: así como las variables sexo (aunque, a este respecto, es de destacar que las mujeres prefieren las variantes más prestigiosas en contextos de formalidad) o edad, combinadas con el registro, no resultan muy operativas, la variable sociocultural sí que lo es, más si se analiza desde una perspectiva diafásica. Las clases menos instruidas prefieren la elisión en registros familiares, y la interdentalización en los formales; la clase media tiende a la interdentalización, aunque -en un registro formal- la solución [k] también puede escucharse; los informantes con un mayor nivel sociocultural son conscientes de que la articulación canónica es la velar oclusiva, que reservan para registros formales, mientras que en un registro familiar prefieren la forma $[\theta]$, aunque el porcentaje de articulación de [k] es también muy relevante.

En definitiva, las diferentes articulaciones de /k/ dentro del grupo /kt/, en función de las diferentes variables (sexo, edad y nivel sociocultural), detectadas y estudiadas en estas 
páginas, certifican la necesidad y la utilidad de los estudios sociolingüísticos a la hora de determinar todos los matices de un proceso de variación lingüística. Esta investigación, tras un detallado análisis, reduce el porcentaje de incidencia de la interdentalización en el mencionado contexto fónico a un $49.7 \%$, frente a los datos más generosos que ofrecían los estudios de geografía lingüística, y muestra las grandes diferencias existentes según el nivel sociocultural del hablante y el registro en el que se produzca la emisión.

\section{NOTAS}

1 El mencionado trabajo de García García de León (2015) sí incluye un estudio acústico de la variación alofónica de /k/ dentro del grupo consonántico /kt/; dentro de este, se le dedican pocas líneas a las fricativas dentales (sordas o, en ocasiones, sonorizadas) por su escasa relevancia en el corpus de la autora.

$2 \quad$ Lo repitió en 1986 (20).

3 El estudio sociolingüístico realizado por este autor solo contó con informantes de entre 18 y 26 años.

4 Un análisis del mapa FON-240, recta, del Atlas Lingüístico (y etnográfico) de CastillaLa Mancha (García Mouton y Moreno Fernández, 2003) muestra los siguientes porcentajes de interdentalización de /k/: Cuenca (93\%), Guadalajara (84\%), Toledo (47\%), Ciudad Real (43\%) y Albacete (25\%); otros mapas, como el de tractor, FON-238, evidencian una incidencia mayor en Albacete, y residual en las provincias de Toledo y Ciudad Real.

5 Cifra ofrecida por el Ayuntamiento de Santander, actualizada el 1 de enero del 2019. Disponible en: https://santander.es/ciudad/santander/cifras.

6 La población inmigrante que reside en la capital de Cantabria es de 12682 personas, lo que sobre una población total de 173721 habitantes arroja un porcentaje del $7 \%$. Esta cifra ha sido extraída del informe del Instituto Cántabro de Estadística, actualizada el 31 de diciembre del 2018. Disponible en: https://www.icane.es/population/population-figures.

7 La población menor de 20 años que reside en la capital de Cantabria es de 27 812; sobre una población total de 173721 habitantes arroja un porcentaje del 14\%. Esta ha sido extraída de los datos ofrecidos por el Ayuntamiento de Santander, actualizados el 1 de enero del 2019. Disponible en: https://santander.es/ciudad/santander/cifras.

$8 \quad$ Porcentajes ofrecidos por el Ayuntamiento de Santander, actualizados el 1 de enero del 2019. Disponible en: https://santander.es/ciudad/santander/cifras.

9 Es decir, se ha agrupado a los informantes en grupos de edad a partir de factores experienciales comunes.

10 Cifras y porcentajes ofrecidos por el Ayuntamiento de Santander, actualizados el 1 de enero del 2019. Disponible en: https://santander.es/ciudad/santander/cifras.

11 Datos ofrecidos por el Ayuntamiento de Santander, actualizados el 1 de enero del 2019. Disponible en: https://santander.es/ciudad/santander/cifras.

12 En el caso de la informante analfabeta, se obvió la parte de las lecturas de la encuesta.

13 R (relajado) y F (formal). 


\section{REFERENCIAS BIBLIOGRÁFICAS}

ALONSO HERNÁNDEZ, C. 1996. "Castilla la Vieja”, en Alvar M. (dir.), Manual de Dialectología Hispánica. El español de España. Barcelona: Ariel, pp. 198-212.

ALVAR, M. 1995. Atlas Lingüístico y Etnográfico de Cantabria. 2 vols. Madrid: Arco/Libros.

ALVAR, M. 1999. Atlas Lingüústico y Etnográfico de Castilla y León. 3 vols. Valladolid: Consejería de Educación y Cultura.

ANTÓN, M. M. 1998. "Del uso sociolingüístico de las oclusivas posnucleares en el español peninsular norteño", Hispania, 81(4), pp. 949-958.

BORREGO NIETO, J. 2001. "El concepto de norma regional y su aplicación a las hablas castellano-leonesas”, en Hernández Alonso, C. (coord.), Actas del II Congreso Internacional de la Lengua Española (Valladolid, 2001). Disponible en: http://congresosdelalengua.es/ valladolid/ponencias/unidad_diversidad_del_espanol/1_la_norma_hispanica/borrego_j. htm.

CEDERGREN, H. 1983. "Sociolingüística”, en López Morales, H. (coord.), Introducción a la lingüística actual. Madrid: Playor, pp. 147-65.

COSERIU, E. 1981. “Los conceptos de 'dialecto', 'nivel' y 'estilo de lengua' y el sentido propio de la dialectología”, Lingüística Española Actual, 3, pp. 1-32.

ECKERT, P. 1997. "Age as a sociolinguistic variable", en Coulmas, F. (ed.), The Handbook of Sociolinguistics. Oxford: Blackwell, pp. 151-167.

FERNÁNDEZ PLANAS, A. M. y MARTÍNEZ CELDRÁN, E. 2008. “Análisis del grupo [kt] y la velocidad del habla a partir de la electropalatografía”, Language Design. Journal of Theoretical and Experimental Linguistics, 1, pp. 63-70.

FERNÁNDEZ JUNCAL, C. 1998. Variación y prestigio: estudio sociolingüístico en el oriente de Cantabria. Madrid: Consejo Superior de Investigaciones Científicas.

FERNÁNDEZ JUNCAL, C. 2000. Neutro de materia y metafonía vocálica en el oriente de Cantabria. Salamanca: Ediciones Universidad de Salamanca.

FERNÁNDEZ JUNCAL, C. 2001. Variación léxica y variación social en el Valle de Aras. Santa Cruz de Tenerife: La Página.

FERNÁNDEZ JUNCAL, C. 2009. "Variación en Cantabria: breve recorrido dialectológico y sociolingüístico”, en Viejo Fernández, X. (coord.), Cien años de Filoloxía Asturiana (19062006). Actes del Congresu Internacional. Oviedo: Universidad de Oviedo-Alvízoras \& Trabe, pp. 177-200.

FERNÁNDEZ-SEVILLA, J. 1990. “Los fonemas implosivos en español”, Thesaurus. Boletín del Instituto Caro y Cuervo, 35(3), pp. 456-505.

FLYDAL, L. 1952. "Remarques sur certains rapports entre le style et l'etat de langue”, Norsk Tidsskrift for Sprogvidenskap, 16, pp. 241-258.

GARCÍA GARCÍA DE LEÓN, C. L. 2015. "Variación en la secuencia /kt/ en español centropeninsular: estudio sincrónico y aplicaciones diacrónicas”, Loquens, 2(1), 1-17.

GARCÍA MOUTON, P. y MORENO FERNÁNDEZ, F. 2003. Atlas Lingüístico (y etnográfico) de Castilla-La Mancha. Disponible en: http://www2.uah.es/alecman. 
GONZÁLEZ FERRERO, J. C. 1986. Sociolingüística y variación dialectal. Estudio del habla de Flores de Aliste. Zamora: Instituto de Estudios Zamoranos "Florián de Ocampo".

LAPESA MELGAR, R. 1981. Historia de la lengua española, Madrid: Gredos.

LABOV, W. 1966. The social stratification of English in New York City. Washington, DC: Center for Applied Linguistics.

LABOV, W. 1972a. Sociolinguistic patterns. Filadelfia: University of Pennsylvania Press.

LABOV, W. 1972b. Language in the inner city. Filadelfia: University of Pennsylvania Press.

LLORENTE MALDONADO DE GUEVARA, A. 1947. Estudios sobre el habla de La Ribera (comarca salmantina ribereña del Duero). Salamanca: Colegio Trilingüe de la Universidad de Salamanca.

LLORENTE MALDONADO DE GUEVARA, A. 1965. “Algunas características lingüísticas de La Rioja en el marco de las hablas del Valle del Ebro y de las comarcas vecinas de Castilla y de Vasconia”, Revista de Fílología Española, 48, pp. 321-350.

LLORENTE MALDONADO DE GUEVARA, A. 1986. El lenguaje estándar español y sus variantes. Salamanca: Instituto de Ciencias de la Educación-Universidad de Salamanca.

LÓPEZ MORALES, H. 1983. Estratificación social del español de San Juan de Puerto Rico. México: Universidad Nacional Autónoma de México.

MARTÍNEZ MARTÍN, F. M. 1983. Fonética y sociolingüística en la ciudad de Burgos. Madrid: Consejo Superior de Investigaciones Científicas.

MORENO FERNÁNDEZ, F. 1996. "Castilla la Nueva”, en Alvar M. (dir.), Manual de dialectología hispánica. El Español de España. Barcelona: Ariel, pp. 213-232.

MORENO FERNÁNDEZ, F. 2009. La lengua española en su geografía. Madrid: Arco/Libros. NAVARRO TOMÁS, T. 1918. Manual de pronunciación española. Madrid: Junta para la Ampliación de Estudios e Investigaciones Científicas, Centro de Estudios Históricos.

NEIRA MARTÍNEZ, J. 1976. El bable. Estructura e historia. Gijón: Ayalga.

PERISSINOTTO, G. S. A. 1975. Fonología del español hablado en la Ciudad de México. Ensayo de un método sociolingüístico. México: El Colegio de México.

QUILIS, A. 1965. "Description phonétique du parler madrilène actuel”, Phonetica, 12, pp. 19-24.

REAL ACADEMIA ESPAÑOLA y ASOCIACIÓN DE ACADEMIAS DE LA LENGUA ESPAÑOLA (RAE y ASALE). 2011. Nueva Gramática de la Lengua Española. Fonética y Fonología. Madrid: Espasa.

SILVA-CORVALÁN, C. 1989. Sociolingüística. Teoría y análisis. Madrid: Alhambra.

SHUY, R. W., WOLFRAM, W. A. y RILEY, W. K. 1968. Field techniques in an urban language study. Washington, DC: Center for Applied Linguistics.

WILLIAMS, L. 1987. Aspectos sociolingüísticos del habla de la ciudad de Valladolid. Valladolid: Secretariado de Publicaciones de la Universidad de Valladolid.

\section{NOTA SOBRE EL AUTOR}

Jaime Peña Arce es investigador posdoctoral en el Departamento de Lengua Española y Teoría de la Literatura, perteneciente a la Universidad Complutense de Madrid, España. ORCID: 00000003-0914-8354 